\title{
VELOCIDADE DA MARCHA E BAIXO DESEMPENHO NA MOBILIDADE FUNCIONAL EM IDOSOS DA ATENÇÃO PRIMÁRIA À SAUDE
}

Conceição da Silva Brito; Universidade Federal do Paraná (UFPR). E-mail: enfbryto@gmail.com; Márcia Marrocos Aristides Barbiero; UFPR; E-mail: marciamarrocos@gmail.com;] Reuber Lima de Sousa. UFPR; E-mail: reuber_souza@hotmail.com; Patrícia Rosa Gonçalves Leta. UFPR. E-mail: patriciarg|@hotmail.com. Kamila Alves Brasileiro. UFPR. E-mail: kamilabrasileiro12@gmail.com. Susanne Elero Betiolli. UFPR. E-mail: susanne.betiolli@gmail.com. Tatiane Prette Kuznier. UFPR. E-mail: tatianeprette@gmail.com. Maria Helena Lenardt. UFPR. E-mail: curitiba.helena@gmail.com.

\section{RESUMO}

Introdução: a velocidade da marcha diminuída e o baixo desempenho da mobilidade podem ocasionar limitações importantes ao idoso, como o declínio no desempenho físico para realizar atividades cotidianas, perda da autonomia e independência. Objetivo: avaliar associação entre velocidade da marcha e baixo desempenho da mobilidade funcional em idosos da Atenção Primária à Saúde. Métodos: estudo de corte transversal desenvolvido em uma Unidade Básica da Saúde em Curitiba/Paraná (Brasil), aprovado por Comitê de Ética em Pesquisa do Setor de Ciências da Saúde sob n²918847. A amostra foi constituída por 389 idosos ( $\geq 60$ anos). Inicialmente foi realizado o rastreio cognitivo dos idosos, e posteriormente aplicado um questionário sociodemográfico e clínico, e avaliados segundo os marcadores do fenótipo da fragilidade e do teste Timed Up and Go. Realizaram-se análises estatísticas descritivas e a distribuição qui-quadrado. Resultados: houve predomínio do sexo feminino $\mathrm{n}=255$ (65,6\%), com idade entre 66 a 70 anos $\mathrm{n}=111(28,5 \%)$, casados $\mathrm{n}=187(48,1 \%)$, com baixa escolaridade $n=138(35,5 \%)$. Relataram ter problemas de saúde $(n=376 ; 96,7 \%)$, com predomínio de doenças cardiovasculares $(\mathrm{n}=261 ; 67,1 \%)$. Dos 389 idosos, predominaram os idosos com baixo desempenho na mobilidade funcional $(\mathrm{n}=330 ; 84,8 \%)$. Apresentaram redução na velocidade da marcha, $81(20,8 \%)$ e destes, $97,5 \%(n=79)$ foi identificado baixo desempenho na mobilidade funcional. Observou-se associação significativa entre redução da velocidade da marcha e baixo desempenho na mobilidade $(p<0,001)$. Conclusão: A associação das variáveis de interesse apontam para a indispensabilidade de estratégias preventivas e gestão de cuidados, para se evitar desfechos negativos nesses idosos.

Palavras-chave: Idoso; Mobilidade funcional; Velocidade da Marcha; Gerontologia. 